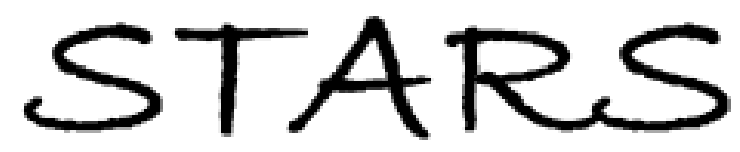

University of Central Florida

STARS

$1-1-2004$

\title{
Broadband optical limiter based on nonlinear photoinduced anisotropy in bacteriorhodopsin film
}

\author{
Yuhua Huang \\ University of Central Florida \\ Georgios Siganakis \\ University of Central Florida \\ M. G. Moharam \\ University of Central Florida \\ Shin-Tson Wu \\ University of Central Florida
}

Find similar works at: https://stars.library.ucf.edu/facultybib2000

University of Central Florida Libraries http://library.ucf.edu

This Article is brought to you for free and open access by the Faculty Bibliography at STARS. It has been accepted for inclusion in Faculty Bibliography 2000s by an authorized administrator of STARS. For more information, please contactSTARS@ucf.edu.

\section{Recommended Citation}

Huang, Yuhua; Siganakis, Georgios; Moharam, M. G.; and Wu, Shin-Tson, "Broadband optical limiter based on nonlinear photoinduced anisotropy in bacteriorhodopsin film" (2004). Faculty Bibliography 2000 s.

4440.

https://stars.library.ucf.edu/facultybib2000/4440

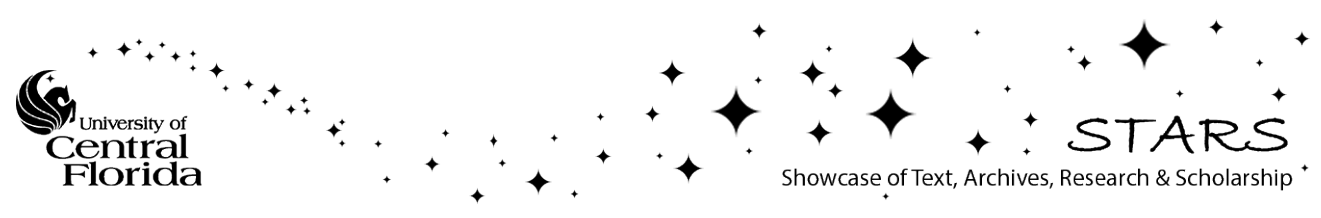




\section{Broadband optical limiter based on nonlinear photoinduced anisotropy in bacteriorhodopsin film}

Cite as: Appl. Phys. Lett. 85, 5445 (2004); https://doi.org/10.1063/1.1828590

Submitted: 10 May 2004 . Accepted: 04 October 2004 . Published Online: 01 December 2004

Yuhua Huang, Georgios Siganakis, M. G. Moharam, and Shin-Tson Wu

ARTICLES YOU MAY BE INTERESTED IN

Broadband thermal optical limiter

Applied Physics Letters 63, 1483 (1993); https://doi.org/10.1063/1.109663

Optical limiter using a lead phthalocyanine

Applied Physics Letters 63, 1880 (1993); https://doi.org/10.1063/1.110635

Nonlinear bleaching, absorption, and scattering of 532-nm-irradiated plasmonic nanoparticles

Journal of Applied Physics 113, 053107 (2013); https://doi.org/10.1063/1.4790798

\section{Applied Physics Letters}

Mid-IR and THz frequency combs special collection

\section{Read Now!}




\title{
Broadband optical limiter based on nonlinear photoinduced anisotropy in bacteriorhodopsin film
}

\author{
Yuhua Huang, Georgios Siganakis, M. G. Moharam, and Shin-Tson Wua) \\ College of Optics and Photonics, University of Central Florida, Orlando, Florida 32816
}

(Received 10 May 2004; accepted 4 October 2004)

\begin{abstract}
Nonlinear photoinduced anisotropy in a bacteriorhodopsin film was theoretically and experimentally investigated and a broadband active optical limiter was demonstrated in the visible spectral range. A diode-pumped second harmonic yttrium aluminum garnet laser was used as a pumping beam and three different wavelengths at $\lambda=442,532$, and $655 \mathrm{~nm}$ from different lasers were used as probing beams. The pump and probe beams overlap at the sample. When the pumping beam is absent, the probing beam cannot transmit the crossed polarizers. With the presence of the pumping beam, a portion of the probing light is detected owing to the photoinduced anisotropy. Due to the optical nonlinearity, the transmitted probing beam intensity is clamped at a certain value, which depends on the wavelength, when the pumping beam intensity exceeds $5 \mathrm{~mW} / \mathrm{mm}^{2}$. Good agreement between theory and experiment is found. (C) 2004 American Institute of Physics. [DOI: 10.1063/1.1828590]
\end{abstract}

An optical power limiter (OPL) is a nonlinear optical device in which its transmittance depends on the incident light intensity. In the low intensity region, the output beam intensity is linearly proportional to the input intensity. However, as the input intensity increases the output intensity gradually saturates. It is a useful device for protecting human eyes and optical sensors from being damaged by lasers. Various materials with large optical nonlinearity and high stability have been extensively investigated for optical limiting applications. ${ }^{1,2}$ In addition to organic and inorganic crystals, biological molecules have also been explored for such applications. Among them, bacteriorhodopsin (bR), a photochromic protein in the purple membrane of halobacterium halobium, has attracted the most attention because of its unique optical properties such as large optical nonlinearity and excellent thermal- and photostabilities. Several techniques, e.g., nonlinear absorption, ${ }^{3}$ self-diffraction, ${ }^{4}$ and nonlinear birefringence, ${ }^{5}$ have been used to demonstrate the optical limiting behavior in a bR film. However, these devices are narrow band. With continuous development of wavelength tunable and high power laser sources, broadband optical power limiters are needed.

Two types of OPL have been developed: passive and active. For a passive OPL, only one laser beam is involved. The transmittance of the OPL decreases as the incident beam intensity increases. However, for an active OPL the device could be controlled by a second beam (pump-probe system) or by an applied voltage. In this letter, we demonstrate a broadband active OPL using the nonlinear photoinduced anisotropy in a bR film. Unlike other approaches, the active OPL based on photoinduced optical anisotropy in bR can be used in the whole visible spectral range. The reason is that photoinduced anisotropy is comprised of photoinduced dichroism predominating around the absorption peak and photoinduced birefringence predominating in the offresonance region. Since bR exhibits a broadband absorption in the visible range, as shown in Fig. 1, its photoinduced anisotropy covers the whole visible region. We theoretically and experimentally investigate the nonlinear performance of

\footnotetext{
${ }^{a)}$ Electronic mail: swu@mail.ucf.edu
}

photoinduced anisotropy in bR at red, green, and blue wavelengths. Good agreement between theory and experiment is obtained.

To understand the photoinduced anisotropy in bR, we need to consider its photophysical processes after the illumination of a linearly polarized light. Initially, molecules are randomly distributed in the bR film, as shown in Fig. 2(a). Upon excitation of a linearly polarized light, those bR molecules (darker color) with the dipole moment aligned close to the polarization direction of the illumination beam are pumped to the excited state and produce photoisomerization from all trans to 13 -cis. ${ }^{7}$ Then the excited molecules go through several spectroscopically distinguished intermediate states $(K, L, M, N$, and $O)$ and the corresponding excited states, and relax back to the initial state spontaneously. ${ }^{7,8}$ Due to the change of molecular structure, the refractive index and absorption coefficient of the bR molecules along the pump beam polarization axis change with the formation of each intermediate state, however, those in the perpendicular direction do not change. Therefore, macroscopic anisotropy of the bR film is induced by the illuminating light. Since the $\mathrm{Br}$ and $M$ states have much longer lifetimes than other intermediate states in the photocycle, we could only consider $\mathrm{Br}$ and $M$ states and their corresponding excited states, ${ }^{9}$ as shown in Fig. 2(b). Here we regard the intermediate $M$ state as $M_{G}$ and the corresponding excited state as $M_{E}$. To analyze

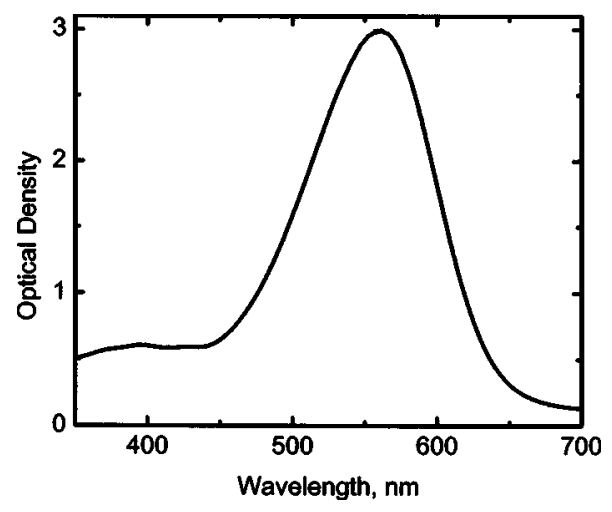

FIG. 1. The optical density of a bR film. $d=\sim 80 \mu \mathrm{m}$. 


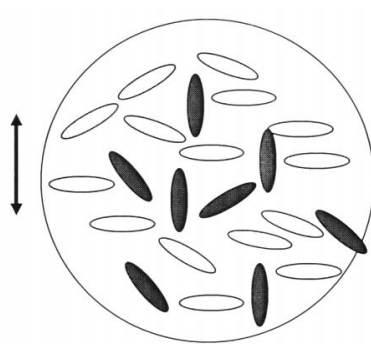

(a)

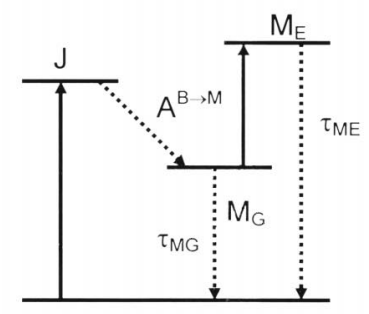

$\mathrm{Br}$

(b)

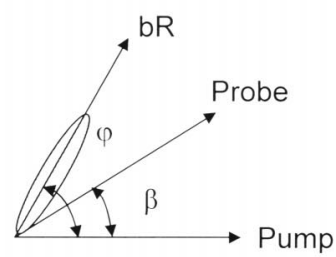

(c)

FIG. 2. An illustration interpreting (a) photoselection of molecules under linearly polarized light, (b) simplified energy-level mode, and (c) angle configuration of the bR molecules and the pumping and probing beams.

the photoinduced anisotropy in bR films, linearly polarized pumping and probing beams are assumed. In Fig. 2(c), we define the polarization direction of the pumping beam as horizontal axis and that of the probing light at an angle $\beta$. For those molecules at angle $\varphi$, the population of a $\mathrm{Br}$ state can be described as follows:

$$
\begin{aligned}
\frac{d N_{\mathrm{Br}}(\varphi)}{d t}= & -\left(\frac{\sigma_{\mathrm{Br}-\text { probe }} A^{B \rightarrow M} I_{\text {probe }}}{h \nu_{\text {probe }}}\right. \\
& \left.+\frac{\sigma_{\mathrm{Br}-\text { pump }} A^{B \rightarrow M} I_{\text {pump }}}{h \nu_{\text {pump }}}\right) N_{\mathrm{Br}}(\varphi) \\
& +\frac{N_{M_{G}}}{\tau_{M_{G}}}+\frac{N_{M_{G}}(\varphi)}{\tau_{M_{E}}} .
\end{aligned}
$$

In Eq. (1), $N_{\mathrm{Br}}(\varphi), N_{M_{G}}(\varphi)$, and $N_{M_{E}}(\varphi)$ are the population density of the $\mathrm{Br}$, ground, and excited $M$ state along $\varphi$ direction, respectively, $h$ is the Planck constant, $\nu_{i}=C / \lambda_{i}$ is the probing and pumping beam frequency, and $A^{B \rightarrow M}=0.64$ is the quantum efficiency for the transition from $\mathrm{Br}$ ground state to the excited state, ${ }^{6} \sigma_{B r-p r o b e}, \sigma_{M-\text { probe }}, \sigma_{B r-p u m p}$, and $\sigma_{M-\text { pump }}$ are the effective absorption cross section of bR's $\mathrm{Br}$ and $M$ states at the probing and pumping wavelengths, respectively. They can be described as follows: ${ }^{10}$

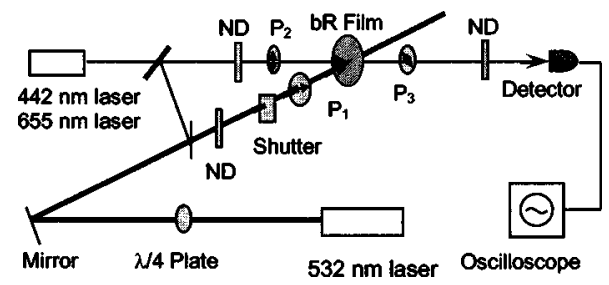

FIG. 3. Experimental apparatus for investigating photoinduced anisotropy in a bR film. $\mathrm{P}_{1}, \mathrm{P}_{2}$, and $\mathrm{P}_{3}$ are polarizers: $\mathrm{P}_{2}$ and $\mathrm{P}_{3}$ are crossed, $\mathrm{P}_{1}$ is at $45^{\circ}$ with respect to $P_{1}$. ND: neutral density filter.

$$
\sigma_{i-j}=\sigma_{i-j}^{ل} \cos ^{2}(\varphi-\beta)+\sigma_{i-j}^{\perp} \sin ^{2}(\varphi-\beta),
$$

where $\sigma_{i-j}^{\|}$and $\sigma_{i-j}^{\perp}$ present the parallel and perpendicular absorption cross section of each state at the probing and pumping wavelengths, respectively, $i$ represents the $\mathrm{Br}$ and $M_{G}$ states, and $j$ represents the probe and pump beams, respectively.

According to the energy-level theory, the total population density $N$ of the bR film and the population density of the $\mathrm{Br}, M_{G}$, and $M_{E}$ states should satisfy the following relationship: $N_{\mathrm{Br}}+N_{M_{G}}+N_{M_{E}}=N$. Similarly, the relationship of $N$ and the population density of each state at angle $\varphi$ should be $N_{\mathrm{Br}}(\varphi)+N_{M_{G}}(\varphi)+N_{M_{E}}(\varphi)=N / 2 \pi$. Since the $M_{E}$ state has a much shorter lifetime than the $\mathrm{Br}$ and $M_{G}$ states, fewer populations can stay in the $M_{E}$ state. As a result, the $M_{E}$ state population can be ignored and the population of the $M_{G}$ state can be simplified as $N_{M_{G}}=N / 2 \pi-N_{\mathrm{Br}}$.

The photoinduced birefringence and dichroism can be generally described as follows:

$$
\begin{aligned}
& \Delta n=\Delta n_{\mathrm{Br}} \int_{0}^{2 \pi} N_{\mathrm{Br}}(\varphi)(\cos \varphi-\sin \varphi) d \varphi \\
& +\Delta n_{M_{G}} \int_{0}^{2 \pi} N_{M_{G}}(\varphi)(\cos \varphi-\sin \varphi) d \varphi, \\
& \Delta D=d \log (e)\left[\left(\sigma_{\mathrm{Br}-\text { probe }}^{\perp}-\sigma_{\mathrm{Br}-\text { probe }}^{\|}\right) \int_{0}^{2 \pi} N_{\mathrm{Br}}(\varphi)\right. \\
& \times\left(\cos ^{2} \varphi-\sin ^{2} \varphi\right) d \varphi+\left(\sigma_{M_{G}-\text { probe }}^{\perp}-\sigma_{M_{G}-\text { probe }}^{\|}\right) \\
& \left.\times \int_{0}^{2 \pi} N_{M_{G}}(\varphi)\left(\cos ^{2} \varphi-\sin ^{2} \varphi\right) d \varphi\right] \text {. }
\end{aligned}
$$

In Eq. (4), $\Delta D$ represents the effective photoinduced dichroism. $\Delta n, \Delta n_{\mathrm{Br}}$, and $\Delta n_{M_{G}}$ present the effective photoinduced birefringence, the photoinduced birefringence per molecule of the $\mathrm{Br}$ and $M_{G}$ states, respectively. Considering the photoinduced dichroism and birefringence, the corresponding transmitted probe beam intensity $I_{\text {out }}$ can be generally written as

$$
\begin{aligned}
I_{\text {out }}= & I_{\text {in }} 10^{-\Delta D} \sin ^{2}(2 \beta) \sin ^{2}\left(\pi \Delta n d / \lambda_{\text {probe }}\right) \\
& \times \exp ^{-\left(\sigma_{\mathrm{Br}-\text { probe }}^{\|} N_{B}+\sigma_{M_{G}}^{\|}-\text {probe }^{N}\right) d},
\end{aligned}
$$

where $I_{\text {in }}$ is the incident probe beam intensity, $\lambda_{\text {probe }}$ is the probing beam wavelength, and $d$ is the film thickness.

Figure 3 shows the experimental apparatus. A linearly polarized diode-pumped Nd-Yttrium aluminum garnet (YAG) laser $(\lambda=532 \mathrm{~nm})$ was converted to circularly polarized light by a quarter-wave plate and then split into two beams by a beam splitter. One of the green beams was used 


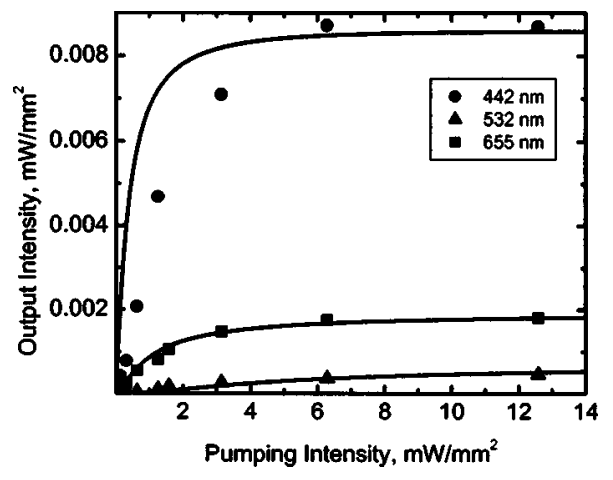

FIG. 4. Pumping beam intensity dependent on transmitted probing beam intensity. Dots are experimental data and solid lines are simulation results using Eq. 10. In our simulation, we use $\sigma_{B}^{\|}=0.1,1.6$, and $0.2 \times 10^{-16} \mathrm{~cm}^{2}$ at $\lambda=655,532$, and $442 \mathrm{~nm}$, respectively; $\sigma_{M}=0,3.8 \times 10^{-18}$, and 0.4 $\times 10^{-16} \mathrm{~cm}^{2}$ at $\lambda=655,532$, and $442 \mathrm{~nm}$, respectively; $\sigma_{i}^{\Downarrow} / \sigma_{i}^{\perp}=10, \tau_{M G}$ $=0.2 \mathrm{~s}$, and $\tau_{M E}=1 \mathrm{~ns}$.

as a pumping beam. A linear polarizer $P_{1}$ was used to select the desired polarization state. The other green beam, a blue He-Cd laser $(\lambda=442 \mathrm{~nm})$, and a red diode laser $(\lambda$ $=655 \mathrm{~nm}$ ) were used as probing beams. The pumping beam overlaps with the probing beam at the bR film which was sandwiched between a pair of crossed Glan-Thompson polarizers $P_{2}$ and $P_{3}$. The polarization direction of the pumping beam selected by $P_{1}$ is inclined at $45^{\circ}$ with respect to $P_{2}$. The bR samples were purchased from Munich Innovative Biomaterials $\mathrm{GmbH}$ (order number WT1N3). Its thickness is $\sim 80 \mu \mathrm{m}$ and optical density is 3 at $\lambda=570 \mathrm{~nm}$.

Without pumping beam, the probing beam could not transmit the analyzer $P_{3}$ because the bR film is isotropic. With the presence of the pumping beam, the analyzer $P_{3}$ transmits a portion of the probing light due to the photoinduced anisotropy in the bR film. The transmitted probing beam intensity highly relies on the pumping beam intensities, as shown in Fig. 4. Figure 4 shows the pumping beam intensity dependent on transmitted probing beam intensity when the probing beam $(\lambda=442,532$, and $655 \mathrm{~nm})$ intensity was set at $0.45,0.28$, and $0.39 \mathrm{~mW} / \mathrm{mm}^{2}$, respectively. In the low pumping intensity regime, the transmitted probing beam intensity increases almost linearly with the increasing pumping beam intensity. However, as the pumping intensity reaches $\sim 5 \mathrm{~mW} / \mathrm{mm}^{2}$, the transmitted probe beam intensity starts to saturate. In the higher intensity regime, the probing beam intensity is clamped to a saturation level. The response time is about $200 \mathrm{~ms}$.

Using the theoretical model described above, we can simulate the experimental results. Based on the absorption spectra published in Ref. 11, we estimated that $\sigma_{B r}^{\|}=0.1,1.6$, and $0.2 \times 10^{-16} \mathrm{~cm}^{2}$, and $\sigma_{M}^{\|}=0,3.8 \times 10^{-18}$, and 0.4 $\times 10^{-16} \mathrm{~cm}^{2}$ at $\lambda=655,532$, and $442 \mathrm{~nm}$, respectively. In our simulations, we assume the dichroic ratio of the $\mathrm{Br}$ and $M$ states is $\sigma_{i}^{\prime \prime} / \sigma_{i}^{\perp}=10$. Varying the dichroic ratio from 10 to 100 does not affect the calculated results noticeably. In addition, from our experiment we found $\tau_{M G}=0.2 \mathrm{~s}$. The lifetime of the excited $M$ state is commonly known to be $\tau_{M E}$ $\sim 1$ ns. $^{6}$ The simulation results are shown as the solid lines in Fig. 4. The agreement between theory and experiment is good.

From Eqs. (3)-(5), the transmitted probe beam intensity is determined by the photoinduced anisotropy which is proportional to the number of the excited bR molecules. Since the number of the excited state molecules is linearly proportional to the pumping beam intensity in the low intensity regime and saturates in the high intensity regime, the transmitted probe beam intensity should have the same trend. Since bR has a larger absorption cross section at $\lambda$ $=532 \mathrm{~nm}$ than that at 442 and $655 \mathrm{~nm}$, the perpendicular transmittance of the probe beam at $\lambda=532 \mathrm{~nm}$ is lower than that at 442 and $655 \mathrm{~nm}$. Equation (5) also indicates that the transmitted probe beam intensity increases with the incident probe beam intensity. Therefore, different applications can be realized at different incident probe beam intensities. For example, by choosing a proper incident probe beam intensity, the device can be used for laser eye protection. This is another advantage in comparison with the previously proposed optical limiters using bR material besides the aforementioned broad bandwidth. Using Eq. (5), we calculated the photoinduced birefringence to be $\Delta n \sim 1.79, \sim 1.3$, and $\sim 4.87$ $\times 10^{-4}$ at $\lambda=655,532$, and $442 \mathrm{~nm}$, respectively. These data are the same order of magnitude as those reported in Refs. 5 and 12 .

For optical limiter applications, the device should be able to respond immediately to the environmental condition. The response time of our sample is slow $(\sim 200 \mathrm{~ms})$ due to the long $M$-state lifetime but it can be improved by changing the temperature, humidity, or $\mathrm{pH}$ value. It is known that the $\mathrm{bR}$ response time in water solution is $\sim 10 \mathrm{~ms}$. Our studies on the temperature effect indicate that the response time decreases with the increasing temperature. At $\sim 50{ }^{\circ} \mathrm{C}$, the bR response time in water solution is reduced to $\sim 3 \mathrm{~ms}$, which is much faster than other organic photochromic materials such as fulgide. ${ }^{13}$

In conclusion, we have investigated the nonlinear photoinduced anisotropy in bR film and demonstrated an active optical limiter based on it. The device can be used in the whole visible region. By choosing a suitable incident probing beam intensity, the device can be used for laser eye protection. The device response time is slow $(\sim 200 \mathrm{~ms})$, which is due to the long lifetime of the $M$ state. The $M$-state lifetime can be significantly reduced to $\sim 3 \mathrm{~ms}$ by changing the temperature, $\mathrm{pH}$ value, and humidity during fabrication process.

This work is supported by DARPA under Contract No. W911NF04C0048.

${ }^{1}$ H. B. Lin, R. J. Tonucci, and A. J. Campillo, Opt. Lett. 23, 94 (1998).

${ }^{2}$ G. Poirier, C. B. de Araujo, Y. Messaddeq, S. J. L. Ribeiro, and M. Poulain, J. Appl. Phys. 91, 10221 (2002).

${ }^{3}$ Q. W. Song, C. Zhang, R. Gross, and R. Birge, Opt. Lett. 18, 775 (1993).

${ }^{4}$ D. N. Rao, C. S. Yelleswarapu, S. R. Kothapalli, D. V. G. L. N. Rao, and B. R. Kimball, Opt. Express 22, 2848 (2003).

${ }^{5}$ G. E. Dovgalenko, M. Klotz, G. J. Salamo, and G. L. Wood, Appl. Phys. Lett. 68, 287 (1996).

${ }^{6}$ Y. H. Huang, S. T. Wu, and Y. Y. Zhao, Appl. Phys. Lett. 84, 2028 (2004).

${ }^{7}$ R. R. Birge, Annu. Rev. Biophys. Bioeng. 10, 315 (1981).

${ }^{8}$ Y. H. Huang, S. T. Wu, and Y. Y. Zhao, Opt. Express 12, 895 (2004).

${ }^{9}$ T. H. Zhang, C. P. Zhang, G. H. Fu, Y. D. Li, L. Q. Gu, G. Y. Zhang, Q. W. Song, B. Parsons, and R. R. Birge, Opt. Eng. (Bellingham) 39, 527 (2000).

${ }^{10}$ E. Y. Korchemskaya, D. A. Stepanchikov, A. B. Druzhko, and T. V. Dyukova, J. Biol. Phys. 24, 201 (1999).

${ }^{11}$ J. A. Stuart, D. L. Mercy, K. L. Wise, and R. R. Birge, Synth. Met. 127, 3 (2002).

${ }^{12}$ Q. W. Song, Y. H. Zhang, R. R. Birge, and J. D. Downie, Proc. SPIE 3470, 226 (1998).

${ }^{13}$ M. Seibold and H. Port, Chem. Phys. Lett. 252, 135 (1996). 